\title{
Recurrent Neural Networks for Narrowband Signal Detection in the Time-Frequency Domain
}

\author{
David Brodrick \\ CSIRO Australia Telescope National Facility, Paul Wild Observatory, \\ Locked Bag 194, Narrabri, NSW 2390, Australia, \\ David.Brodrick@csiro.au \\ Douglas Taylor \\ School of Computer Science and Software Engineering, Queensland \\ University of Technology, GPO Box 2434, Brisbane, QLD 4001, \\ Australia,di.taylor@qut.edu.au \\ Joachim Diederich \\ School of Information Technology and Electrical Engineering, University \\ of Queensland, St Lucia, QLD 4072, Australia, joachimd@itee.uq.edu.au
}

\begin{abstract}
A recurrent neural network was trained to detect the timefrequency domain signature of narrowband radio signals against a background of astronomical noise. The objective was to investigate the use of recurrent networks for signal detection in the Search for Extra-Terrestrial Intelligence, though the problem is closely analogous to the detection of some classes of Radio Frequency Interference in radio astronomy.
\end{abstract}

\section{Description}

Artificial neural network based data processing has taken inspiration from biological neural networks, such as the brain. An artificial neural network consists of simple units (the "neurons") with weighted connections between them (comparable to biological "synapses"). During network training, the connection weights are tuned to encode a distributed map between the input space and target output. Network behaviour is customised to a particular problem domain by selecting training examples with corresponding target responses and an appropriate network architecture.

An architecture with recurrent connections allows the network to maintain state and enables the processing of temporal sequences. The partially recurrent network architecture used in this experiment was an Elman network with ten inputs, ten hidden and context units and a single output unit (Elman 1990).

In this experiment, radio-spectrometer data was used as input and the network was trained to identify sequences containing the signature of a narrowband radio signal. The target output was 1.0 at the end of a sequence containing a signal, and 0.0 when no signal was present. A threshold of 0.85 was used as 
the decision limit during network evaluation. The learning rate was 0.005 with momentum 0.9.

Each input sequence comprised fifteen consecutive integration periods of ten spectral channels generated from SETI@home data from the Arecibo Observatory (Sullivan et al. 1996). Monochromatic signals were generated at a variety of power levels and digitally combined with half of the astronomical data sequences. In all, 500 sequences were used for training.

After each complete iteration of the training set, the network was evaluated on a validation set of 250 sequences and the RMS error was recorded. The network weights with minimum error on the validation set were selected for evaluation of system performance. The use of a validation set serves to prevent the network from over-learning the training data.

The RMS error on the validation data set reached a minimum after 300 repetitions of the training set. When evaluated on a test data set of 250 sequences this network produced 8 false positive and 11 false negative classifications $(7.6 \%$ incorrect). Analysis of the false negative responses indicated that these were preferentially associated with signals having low power with respect to the radio noise level.

\section{Discussion}

In this experiment, the motivation was to evaluate the network using data relevant to the Search for Extra-Terrestrial Intelligence (SETI). The narrowband signals were generated with characteristics to simulate one possible signature of a radio beacon from an extra-terrestrial civilisation. The signals had bounded random frequency drift rates to mimic uncompensated Doppler drift. The signals also had Gaussian power profiles to match the transit of a point source across the beam of the Arecibo telescope. Figure 1 shows examples of input sequences with and without narrowband signals injected.

The sophisticated instruments used in modern SETI surveys produce vast amounts of data for analysis. Mining this data for candidate signals forms a data processing bottleneck for such surveys and, for instance, has spawned the SETI@home project to utilise millions of computers for the task (Sullivan et al. 1996). This experiment and other work suggests neural networks may offer a viable alternative to other data mining techniques (Taylor et al. 1999). Neural networks are computationally efficient and can be realised in software or hardware implementations. A limitation of neural networks for SETI is that assumptions must be made about the likely characteristics of signals from extraterrestrial civilisations in order to generate training data.

Automated detection of narrowband signals is also relevant to general radio astronomy where narrowband Radio Frequency Interference (RFI) from electronic equipment, satellites, etc. can corrupt the astronomical interpretation of data. Traditional RFI detection techniques can require prior knowledge of the receiver system and/or interference source. However RFI characteristics vary between different instruments, frequencies and observations and effective RFI rejection can require laborious manual intervention.

A neural network solution requires no explicitly defined model because network training can be accomplished using previously recorded examples of RFI. 

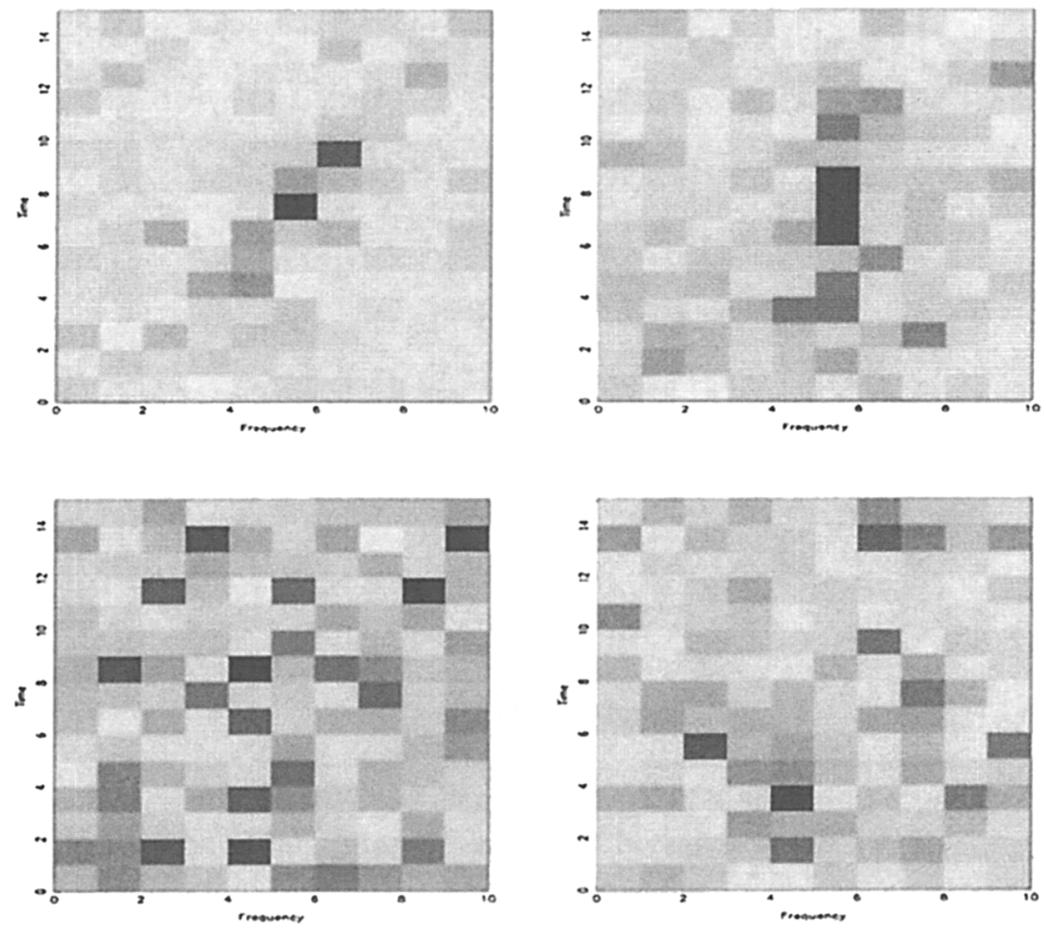

Figure 1. Time-frequency domain input sequences. The top panels show sequences containing narrowband signals while the bottom two panels show radio noise only.

This helps to minimise system complexity while maximising the ability of the network to perform the required task of identifying RFI peturbed data.

\section{Conclusion}

Recurrent neural networks are an efficient solution to a broad range of sequence processing and classification tasks. This experiment has demonstrated potential for Elman networks at detecting the presence of narrowband signals in noisy time-frequency radio data. Further development may provide a robust and effcient tool for use in the Search for Extra-Terrestrial Intelligence and astronomical interference mitigation systems.

\section{References}

Elman, J. 1990, Cognitive Science, 14, 179

Sullivan, W., Werthimer, D., Bowyer, S., Cobb, D., Gedye, J., \& Anderson, D. 1996, in Proceedings of the 5th International Conference on Bioastronomy

Taylor, D., Towsey, M., Finn, G., \& Diederich, J. 1999, in Proceedings of the 6th International Conference on Bioastronomy 


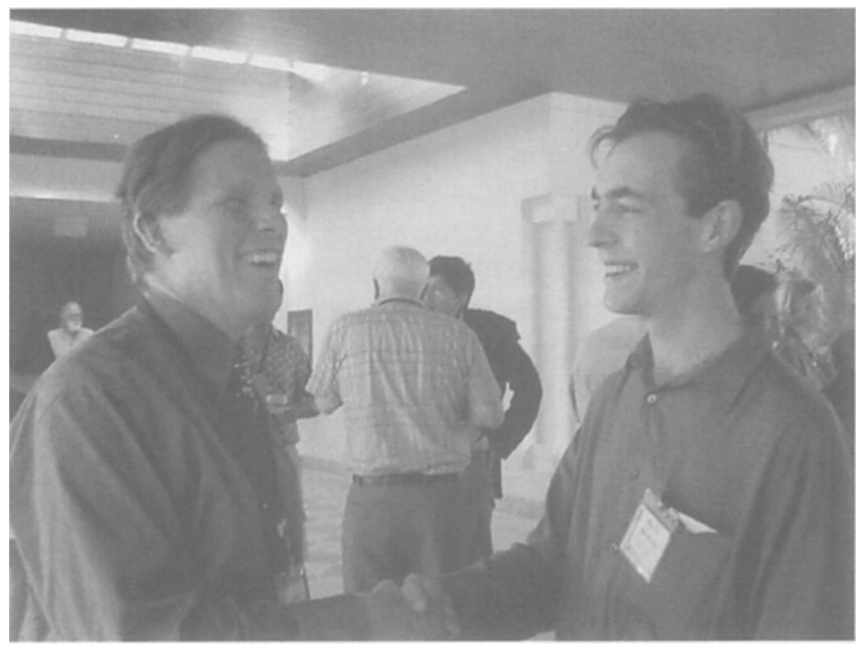

Kent Cullers \& David Broderick (photo: Seth Shostak)

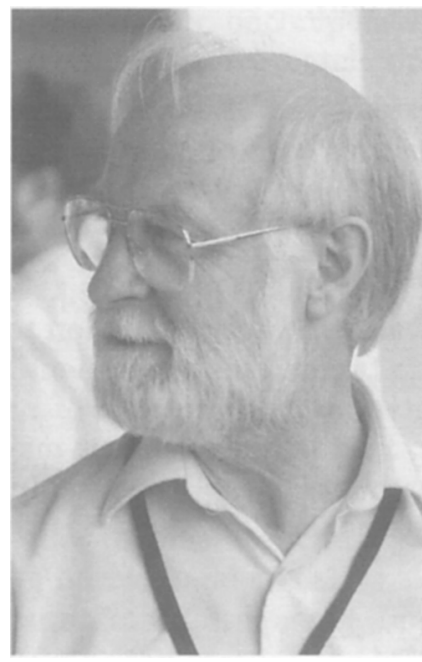

Ron Ekers (photo: Seth Shostak) 